\title{
Metabolismo posprandial en adultos mayores normales de nivel del mar
}

\author{
FAUSTO GARMENDIA ${ }^{1,2}$, ROSA PANDO ${ }^{1,2}$, WILLIAM TORRES ${ }^{1,2}$, WUILI VALQUI ${ }^{1,2}$, \\ CARLOS JAMIESON ${ }^{3}$, NATHAN BLUFSTEIN ${ }^{3}$ \\ ${ }^{1}$ Instituto de Investigaciones Clínicas, UNMSM. ${ }^{2}$ Hospital Nacional Dos de Mayo. ${ }^{3}$ Laboratorio Blufstein.
}

\begin{abstract}
RESUMEN
OBJETIVO: Determinar las modificaciones normales del metabolismo después de la ingestión de alimentos. MATERIAL Y MÉTODOS: Se ha estudiado a 33 personas de ambos géneros, adultos mayores, de nivel del mar, tanto en ayunas como después de la ingesta de una mezcla alimenticia consistente en $730 \mathrm{kcal}$, con $55,4 \%$ de grasas, $37,2 \%$ de hidratos de carbono y 7,4\% de proteínas. Se ha medido el perfil metabólico en ayunas y luego de la ingesta alimentaria, la glucosa, triglicérido (Tg), ácidos grasos no esterificados (AGNE), insulina por métodos convencionales hasta la $6^{\text {a }}$ hora; se ha calculado las concentraciones de colesterol LDL y VLDL utilizando la fórmula de Friedewald; el colesterol no-HDL, mediante la sustracción del valor del colesterol HDL al valor de colesterol total (CT). RESULTADOS: La glicemia se elevó discretamente, regresando a valores basales a la $2^{\text {a }}$ hora; la insulinemia se incrementó durante 4 horas; los triglicéridos comenzaron a elevarse en sangre a partir de la $2^{\mathrm{a}}$ hora y llegaron al máximo a la $4^{\mathrm{a}}$ hora, para luego iniciar el descenso no completo en la $6^{a}$ hora. Los AGNE disminuyeron a partir de los 30 minutos, llegando al máximo de caída a las 2 horas; luego se apreció un rebote máximo a la $6^{\mathrm{a}}$ hora, inclusive mayor al valor basal. CONCLUSIONES: Estos resultados demuestran que no es suficiente obtener información del metabolismo intermediario en ayunas, sino que el estudio posprandial permite mayor información. Es la primera vez en nuestro país que se obtiene información sobre el metabolismo intermediario en fase posprandial en una muestra de la población normal que se encuentra en una edad de mayor riesgo cardiovascular.
\end{abstract}

Palabras clave: Metabolismo; ingestación de alimentos; población urbana; costas; ayuno.

\section{POSPRANDIAL METABOLISM IN OLDER ADULTS AT SEA LEVEL SUMMARY}

OBJETIVE: To determine normal metabolism variations following food ingestion. MATERIAL AND METHODS: A total of 33 normal middle-aged and old adults, 16 female and 17 male, 45-70 years old (average 56,3 $\pm 7,32$ ), were studied both after a 12-14 hours nocturnal fast and following ingestion of a food mixture containing $730 \mathrm{kcal}, 55,4 \%$ fat, 37,2\% carbohydrates and 7,4\% protein, an almost standard breakfast. Blood samples were obtained at fasting state and during 6 hours after the ingestion of the meal described before. Glucose, triglycerides, total cholesterol and HDL cholesterol were measured by enzymatic methods, insuline by radioimmune assay and non-esterified fatty acids (NEFA) by the method of Duncombe. RESULTS: Blood glucose rose from 30 minutes to the $2^{\text {nd }}$ hour; insulin from 30 minutes to the $4^{\text {th }}$ hour, triglycerides rose from the $2^{\text {nd }}$ to $4^{\text {th }}$ hour and then began to decline at the $6^{\text {th }}$ hour without achieving basal value, NEFA diminished from 30 minutes to the $2^{\text {nd }}$ hour and then a rebound was observed even higher than the basal terms at the $6^{\text {th }}$ hour. CONCLUSIONS: The results obtained are considered as a normal pattern for middle-aged and old persons living at sea level and demonstrate that a posprandial study gives a greater information about the metabolism than the carried out only at basal state.

Key words: Metabolism; eating; urban population; coasts; fasting.

\section{Correspondencia:}

Dr. Fausto Garmendia Lorena

Facultad de Medicina, UNMSM

Av. Grau 755. Lima 1 - Perú

E-mail: garmendial@terra.com.pe 


\section{INTRODUCCIÓN}

En el momento actual existe una corriente de investigación dirigida a estudiar las variaciones de los lípidos durante el período posprandial, basada en la idea que en estas condiciones habría una mejor correlación entre los resultados de los lípidos y la aparición de eventos cardiovasculares (ECV), que cuando uno mide los lípidos en ayunas, habida cuenta que las personas no están en un estado permanente de ayuno y mas bien ingieren alimentos varias veces al día. Por otro lado, permite conocer las fluctuaciones de otros metabolitos, como la glucosa, insulina y ácidos grasos no esterificados (AGNE).

Cuando uno estudia el metabolismo posprandial, está midiendo principalmente las variaciones de las lipoproteínas ricas en triglicéridos (Tg) y de los AGNE. Los Tg tienen 2 orígenes, los quilomicrones que contienen $\mathrm{Tg}$ de origen exógeno y las VLDL producidas por el hígado, cuya participación es variable de acuerdo al estado metabólico en el que se encuentre una persona. La forma más exacta de diferenciarlos es mediante la ultracentrifugación diferencial cuantitativa, debido a que son partículas de tamaño y densidad diferentes; sin embargo, esta metodología es complicada y onerosa. Los quilomicrones son lipoproteínas grandes y muy poco densas, que son transportadas por la apoproteína B48, en tanto que las VLDL son más pequeñas, más densas, con un contenido mayor de colesterol y son transportadas por la apoproteína B 100 . Se puede utilizar la adición de vitamina A en la alimentación para medirlas por métodos bioquímicos; esta vitamina aparece con los quilomicrones, que pueden entonces ser medidos como retinil palmitato.

En el momento actual no se cuenta con una prueba de sobrecarga alimenticia estándar; lo cual entorpece la evaluación de los resultados obtenidos por diferentes autores.

Pese a la diversidad de resultados, los aspectos generales más aceptados son los siguientes:
1. Tanto más alta sea la cifra basal de $\mathrm{Tg}$, la respuesta a la sobrecarga es mayor.

2. Tanto mayor sea el contenido de lípidos de la sobrecarga, más alta es la curva; con $5 \mathrm{~g}$ de grasa, la respuesta es nula. Se ha utilizado sobrecargas de 5 a 90 g y cada vez la respuesta ha sido mayor $\left({ }^{1}\right)$.

3. El tiempo de observación después de la ingestión alimenticia es así mismo variable. La mayoría utiliza un tiempo de 6 a 8 horas $\left({ }^{1-5}\right)$.

4. La adición de otros nutrientes, como carbohidratos, proteínas y fibra modifica la magnitud de la respuesta $\left({ }^{2-4}\right)$.

5. El pico de elevación de los Tg se ha encontrado entre 2 a 6 horas; depende del contenido de grasas y carbohidratos.

6. El contenido del tipo de ácidos grasos de la sobrecarga modifica la respuesta. Los ácidos grasos saturados producen una mayor elevación de triglicéridos.

7. El ejercicio disminuye la respuesta lipémica posprandial $\left({ }^{5}\right)$.

Hemos tomado en consideración todos estos hechos y, además, nos hemos ubicado en un nivel más fisiológico para utilizar la técnica de sobrecarga alimenticia del presente trabajo, que en nuestro conocimiento es la primera vez que se realiza en nuestro país.

\section{MATERIAL Y MÉTODOS}

Se ha estudiado a 33 personas presuntas normales del nivel del mar (Lima, $150 \mathrm{~m}$ sobre el nivel del mar), 16 mujeres y 17 varones, de 41 a 72 años, promedio $56,3 \pm 7,32$ años; todas las mujeres eran posmenopáusicas y no recibían tratamiento hormonal de reemplazo, cuyas características aparecen en la Tabla 1.

Se ha utilizado los siguientes criterios de inclusión: Personas aparentemente normales de 40 a 70 años de edad, de ambos géneros, con el índice de masa corporal (IMC) menor de 27, circunferencia abdominal (CA) $<100 \mathrm{~cm}$ en varones y $<90 \mathrm{~cm}$ en mujeres, glicemia en ayu- 
Tabla 1.- Características de la muestra.

\begin{tabular}{lcrl}
\hline \multicolumn{1}{c}{$\mathrm{n}$} & \multicolumn{1}{c}{$\begin{array}{c}\text { Varones } \\
17\end{array}$} & $\begin{array}{c}\text { Mujeres } \\
13\end{array}$ & $p$ \\
\hline Edad, años & $56,4 \pm 1,82^{*}$ & $56,3 \pm 1,13$ & 0,92 \\
Peso, kg & $64,7 \pm 1,56$ & $56,1 \pm 1,13$ & 0,0001 \\
Talla, m & $1,61 \pm 0,01$ & $1,53 \pm 0,01$ & 0,0001 \\
IMC, kg/m2 & $24,7 \pm 0,46$ & $23,6 \pm 0,43$ & 0,11 \\
PAs, mmHg & $122,9 \pm 1,82$ & $113,1 \pm 2,00$ & 0,001 \\
PAd, mmHg & $73,5 \pm 1,70$ & $70,7 \pm 1,78$ & 0,27 \\
Pulso, minuto & $73,0 \pm 2,00$ & $68,8 \pm 1,51$ & 0,12 \\
CA, cm & $91,6 \pm 1,32$ & $79,6 \pm 2,27$ & 0,0001 \\
& & & \\
\hline
\end{tabular}

* Promedio \pm EMS, IMC = Índice de masa corporal, $P A s=$ Presión arterial sistólica,

$P A d=$ presión arterial diastólica,

$C A=$ Circunferencia abdominal

nas $<110 \mathrm{mg} / \mathrm{dL}$, colesterol total $<240 \mathrm{mg} /$ dL, colesterol LDL $<160 \mathrm{mg} / \mathrm{dL}$, triglicéridos $<250 \mathrm{mg} / \mathrm{dL}$.

Los criterios de exclusión fueron: Diagnóstico de hipertensión arterial, diabetes mellitus, dislipoproteinemias, obesidad, enfermedades tiroideas, hepáticas, enfermedades cardiovasculares, renales, síndrome de mala absorción intestinal, antecedentes familiares de diabetes mellitus, hipertensión arterial y dislipoproteinemia. Presión arterial >140/90, colesterol total $>240 \mathrm{mg} / \mathrm{dL}$, colesterol LDL superior a $160 \mathrm{mg} / \mathrm{dL}$, colesterol No-HDL > 190 $\mathrm{mg} / \mathrm{dL}$ o $\mathrm{Tg}>250 \mathrm{mg} / \mathrm{dL}$.

Después de un ayuno nocturno de 12 a 14 horas, se les tomó una muestra basal de sangre y luego ingirieron un desayuno consistente en $80 \mathrm{~g}$ de pan, $30 \mathrm{~g}$ de mantequilla y 1 huevo con una infusión de té o de manzanilla con $10 \mathrm{~g}$ de azúcar, que contiene $730 \mathrm{kcal}, 45 \mathrm{~g}$ de grasa (55,4\% del valor calórico total), $68 \mathrm{~g}$ de carbohidratos $(37,2 \%), 13,4 \mathrm{~g}$ de proteínas $(7,34 \%)$; posteriormente se tomó muestras de sangre a los 30, 60, 120, 240 y 360 minutos después de iniciada la ingesta alimenticia.

Se ha medido glucosa, triglicéridos ( $\mathrm{Tg}$ ), colesterol total (CT) y el colesterol HDL, por métodos enzimáticos (Merck, Darmstadt), insulina por radioinmunoensayo (Diagnostic Products, Davis, California); los ácidos grasos no esterificados por el método de Duncombe $\left({ }^{6}\right)$. En la muestra basal se ha calculado las fracciones LDL y VLDL utilizando la fórmula de Friedewald. El colesterol No-HDL se calculó restando el valor del colesterol HDL a la cifra del colesterol total.

Para los cálculos estadísticos de los promedios de hombres vs. mujeres se ha empleado la prueba " $t$ " de Student.

\section{RESULTADOS}

En la comparación por géneros, los varones tuvieron un peso y talla mayores que las mujeres, pero el IMC fue semejante; la presión arterial sistólica fue ligeramente superior en los varones, dentro de los límites normales. No se encontró diferencias en la edad, IMC, concentraciones de glucosa, Tg, insulina, AGNE, perfil lipídico, por lo que los cálculos estadísticos subsecuentes han sido efectuados reuniendo los valores de hombres y de mujeres.

En la Tabla 2 se muestra los resultados del perfil metabólico en ayunas, que corresponde a un rango para personas de la edad correspondiente. Tres personas del sexo femenino fueron eliminadas para los cálculos estadísticos, por tener colesterol LDL superior a $160 \mathrm{mg} / \mathrm{dL}$,

Tabla 2.- Perfil metabólico basal.

\begin{tabular}{lrrc}
\hline \multicolumn{1}{c}{$\mathrm{n}$} & \multicolumn{1}{c}{ Varones } & \multicolumn{1}{c}{ Mujeres } & \multicolumn{1}{c}{$p$} \\
\hline Glucosa mg/dL & $82,7 \pm 3,52 *$ & $82,0 \pm 3,47$ & 0,89 \\
Insulina $\mu \mathrm{UI} / \mathrm{mL}$ & $9,68 \pm 0,95$ & $9,23 \pm 0,91$ & 0,92 \\
Tg, mg/dL & $125,9 \pm 12,7$ & $125,3 \pm 13,1$ & 0,97 \\
AGNE, $\mu$ Eq/L & $509,7 \pm 44,9$ & $561,5 \pm 53,2$ & 0,46 \\
CT, mg/dL & $179,3 \pm 6,01$ & $196,2 \pm 9,02$ & 0,11 \\
HDL, mg/dL & $35,1 \pm 1,75$ & $41,3 \pm 3,92$ & 0,12 \\
VLDL, mg/dL & $27,9 \pm 4,83$ & $25,1 \pm 2,63$ & 0,64 \\
No-HDL, mg/dL & $144,3 \pm 5,39$ & $154,0 \pm 6,74$ & 0,26 \\
\hline
\end{tabular}

* Promedio \pm EMS (error estandar de la media) 
colesterol No-HDL $>190 \mathrm{mg} / \mathrm{dL}$ o $\mathrm{Tg}>250$ $\mathrm{mg} / \mathrm{dL}$.

Luego de la ingesta alimenticia, la glicemia se elevó en una forma menos pronunciada que cuando se administra glucosa pura, llegando al máximo entre los 30 a 60 minutos; en ningún caso superó $154 \mathrm{mg} / \mathrm{dL}$; a las 2 horas, la glicemia en todos los casos permaneció debajo de $126 \mathrm{mg} / \mathrm{dL}$ (Tabla 3).

La concentración de Tg muestra inicialmente un ligero descenso durante la primera hora posprandial, para luego elevarse y llegar al máximo en la $4^{a}$ hora, después de lo cual se observa descenso en la $6^{a}$ hora, que no llegó al nivel de los valores basales.

La concentración máxima de insulina se observó a los 30 minutos, que coincide con el pico de glucosa. La insulinemia se mantuvo alta hasta la $4^{\text {a }}$ hora, que responde a los valores tanto de glicemia como de los triglicéridos.

Los AGNE mostraron una movilización inmediata; desde los 30 descendieron significativamente hasta llegar al valor más bajo a los 120 minutos; luego se observa un repunte, que inclusive supera el valor basal a la $6^{\text {a }}$ hora.

\section{DISCUSIÓN}

Los resultados de esta investigación muestran las modificaciones metabólicas que ocurren en un grupo de personas normales mayores de 40 años de edad, de ambos géneros. Se aprecia una elevación moderada de la glicemia, menor a la que se suele observar cuando se administra una sobrecarga de glucosa y que está vinculada al hecho de tratarse de una mezcla de nutrientes $\left({ }^{3}\right)$. Las concentraciones de insulina corresponden a las elevaciones de la glicemia, desde que la glucosa es un estimulante de la secreción pancreática de insulina. Por otro lado, en normales, se observa que las concentraciones de Tg se elevan recién después de una hora de la ingesta de alimentos y llegan al máximo en la $4^{\mathrm{a}}$ hora, para luego descender lentamente; a la ${ }^{\text {6a }}$ hora están aún por encima de lo basal.

Esta prueba, tal como la estamos estandarizando, servirá de patrón para estudiar otros grupos de pacientes con factor de riesgo cardiovascular, como son los obesos, diabéticos, hipertensos, con alteraciones primarias de los lípidos y otras condiciones similares ${ }^{7}$ ).

Se ha investigado el comportamiento lipídico posprandial en sujetos normales parientes de personas que a edad temprana tuvieron enfermedad arterial coronaria $\left({ }^{8}\right)$. Los descendientes con antecedentes de enfermedad cardiaca coronaria de edad superior a los 40 años tuvieron una elevación mayor que los descendientes con antecedentes familiares pero de edad menor, lo que hace ver que la edad es importante, además del antecedente familiar.

El ejercicio es una actividad importante para evitar el desarrollo de enfermedades como la obesidad y la diabetes mellitus y además tiene un efecto favorable en la regulación del metabolismo lipídico $\left.{ }^{5,9}\right)$, por lo que será muy va-

Tabla 3.- Glucosa, triglicéridos, insulina y AGNE basales en el período posprandial $(\mathrm{n}=30)$.

\begin{tabular}{lrrrrrr}
\hline & \multicolumn{1}{c}{0} & \multicolumn{1}{c}{30} & \multicolumn{1}{c}{60} & \multicolumn{1}{c}{120} & \multicolumn{1}{c}{240} & 360 \\
\hline Glucosa mg/dL & $82,4 \pm 2,46$ & $115,0 \pm 3,77$ & $95,8 \pm 4,83$ & $88,7 \pm 3,24$ & $87,3 \pm 2,31$ & $88,6 \pm 1,70$ \\
Tg mg/dL & $125,6 \pm 9,03$ & $120,3 \pm 8,11$ & $123,6 \pm 9,43$ & $148,0 \pm 10,8$ & $196,6 \pm 15,0$ & $171,7 \pm 14,6$ \\
Insulina $\mu \mathrm{UI} / \mathrm{mL}$ & $9,43 \pm 1,06$ & $51,9 \pm 4,94$ & $44,6 \pm 5,96$ & $30,7 \pm 3,10$ & $15,8 \pm 1,96$ & $8,33 \pm 0,86$ \\
AGNE $\mu \mathrm{Eq} / \mathrm{L}$ & $532,1 \pm 34,1$ & $358,7 \pm 22,6$ & $224,7 \pm 19,8$ & $215,3 \pm 17,0$ & $329,5 \pm 18,6$ & $638,6 \pm 34,9$ \\
\hline
\end{tabular}

Promedio $\pm E S M$ 
lioso estudiar el metabolismo posprandial en condiciones de actividad física de diversa magnitud.

Por lo anteriormente expuesto, consideramos que el estudio posprandial del metabolismo, particularmente lipídico, permitirá en el futuro tener una apreciación mucho mejor y más temprana para el diagnóstico y tratamiento de los trastornos metabólicos innatos o adquiridos, que la simple determinación basal.

Por otro lado, el esquema de sobrecarga alimenticia que se propone intenta inducir a efectuar una prueba más fisiológica, que en el futuro pueda ser considerada como una prueba estándar.

Estudio realizado con financiamiento del Fondo de Desarrollo Universitario (FEDU), UNMSM, 1998.

\section{BIBLIOGRAFÍA}

1. Dubois C, Beaumier G, Juhel C, Armand M, Portugal H, Pauli AM, et al. Effects of graded amounts (0-50g) of dietary fat on postprandial lipemia and lipoproteins in normolipemic adults. Am J Clin Nutr 1998; 67: 31-8.
2. Jeppesen J, Chen YI, Zhou MY, Schaaf P, Coulston A, Reaven GM. Postprandial triglyceride and retinyl ester responses to oral fat: effects of fructose. Am J Clin Nutr 1995; 61: 787-91.

3. Jeppesen J, Chen YD, Zhou MY, Wang T, Reaven GM. Effect of variations in oral fat and carbohydrate load on postprandial lipemia. Am J Clin Nutr 1995; 62: 1201-5.

4. Dubois C, Armand M, Senft M, Portugal H, Pauli AM, Bernard PM, et al. Chronic oat bran intake alters postprandial lipemia and lipoproteins in healthy adults. Am J Clin Nutr 1995; 61: 325-33.

5. Hardman A, Lawrence JEM, Herd SL. Postprandial lipemia in endurance trained people during a short interruption to training. J Appl Physiol 1998; 84 (6): 1895-901.

6. Duncombe WG. The colorimetric micro determination of non esterified fatty acids in plasma. Clin Chim Acta 1964; 9: 122.

7. Weintraub MS, Eisenberg S, Breslon JL. Different patterns of postprandial lipoprotein metabolism in normal, type II, type III and type IV hyperlipoproteinemic individuals. Effects of treatment with cholesteramine and genfibrozil. J Clin Invest 1987; 79: 1110-9.

8. Slyper AH, Zvereva S, Schectman G, Hoffmann RG, Pleuss J, Walker JA. Normal postprandial lipemia and chylomicron clearance in offspring of parents with early coronary artery disease. J Clin Endocrinol Metab 1998; 83: 1106-13.

9. Weintraub MS, Rosen Y, Otto R, Eisenberg S, Breslow JL. Physical exercise conditioning in absence of weight loss reduce fasting and postprandial triglyceride-rich lipoprotein levels. Circulation 1989; 79: 1007-14. 